Case Report

\title{
Pneumomediastinum and Mediastinal Hematoma Secondary to Right Brachiocephalic Vein Thrombectomy Mimicking STEMI
}

\author{
Prem Shukla, Prudence Dy, Rishi Raj, \\ Sayee Sundar Alagusundaramoorthy, and Noel Nivera \\ Department of Medicine, Monmouth Medical Center, Long Branch, NJ 07740, USA \\ Correspondence should be addressed to Prudence Dy; prudencekhody@gmail.com
}

Received 12 April 2017; Revised 4 June 2017; Accepted 20 June 2017; Published 18 July 2017

Academic Editor: Kjell Nikus

Copyright (C) 2017 Prem Shukla et al. This is an open access article distributed under the Creative Commons Attribution License, which permits unrestricted use, distribution, and reproduction in any medium, provided the original work is properly cited.

\begin{abstract}
A 50-year-old male with a history of hemodialysis dependent chronic kidney disease presented to our emergency department with acute midsternal crushing chest pain. Patient was diagnosed with acute anterolateral wall Myocardial Infraction due to the presence of corresponding ST segment elevations in EKG and underwent emergent cardiac catheterization which revealed normal patent coronaries without any disease. He continued to have chest pain for which CT of the chest was done which revealed pneumomediastinum with mediastinal hematoma, due to the recent attempted thrombectomy for thrombus in his right brachiocephalic vein.
\end{abstract}

\section{Background}

It is absolutely imperative to rule out cardiac ischemia in patients who present with chest pain and ST segment elevation on EKG. Emergent cardiac catheterization is the standard of care across all hospitals in the United States. However, once a cardiac etiology is ruled out other diagnosis should be sought. Around $26 \%$ of angiography done in acute phase of suspected STEMI has been shown to be normal [1]. The incidence of alternative conditions mimicking STEMI is around 2.3\% [2]. Pneumomediastinum is a rare condition that can mimic STEMI. This case report aims to report pneumomediastinum after unsuccessful right brachiocephalic vein thrombectomy mimicking a STEMI.

\section{Case Presentation}

We present the case of a 50-year-old male with a history of hemodialysis dependent end-stage renal disease, HIV on antiretroviral therapy, hypertension, and hyperlipidaemia who presented to our emergency room with stinging nonradiating midsternal chest pain since the afternoon associated with difficulty in breathing. Patient was a former smoker with 29-pack-year smoking history and a former alcoholic.
Family history revealed MI in the father at the age of 56 . Patient underwent unsuccessful right brachiocephalic vein thrombectomy a day prior to presentation to the emergency room.

At the time of physical examination, blood pressure was $128 / 97 \mathrm{mmHg}$, heart rate was $84 /$ minute, respiratory rate was $15 /$ minute, temperature was $99.7 \mathrm{~F}$, and $\mathrm{SpO}_{2}$ was $93 \%$ in room air. Patient was a moderately built obese male, alert, diaphoretic, and in moderate distress from his chest pain and shortness of breath. Cardiovascular examination revealed normal cardiac sounds, no murmurs/rubs, and no chest wall tenderness. Breath sounds were heard in all lung fields without any added sounds. Peripheral pulses were equal and no delay was noted. His AV Fistula in the right arm had a good palpable thrill. The rest of the exam was unremarkable. There were no signs of brachiocephalic vein thrombosis.

\section{Investigations}

Complete blood count with differential showed normocytic normochromic anemia with haemoglobin of $13.2 \mathrm{mg} / \mathrm{dl}$ (normal range $13.5-18 \mathrm{mg} / \mathrm{dl}$ ), white cell count of $8800 / \mathrm{cu} \mathrm{mm}$ (normal range $4500-11000 / \mathrm{cumm}$ ), and platelets of $253,000 / \mathrm{cu} \mathrm{mm}$ (normal range 140,000-450,000/cu mm). 


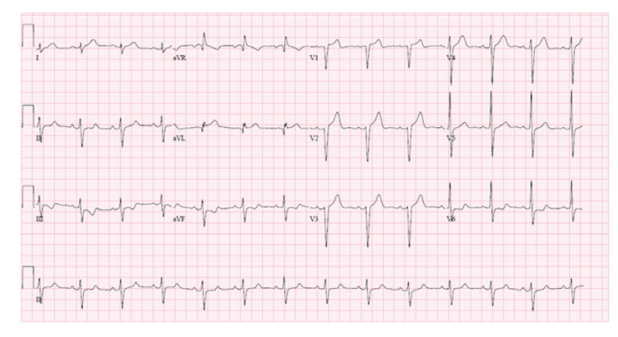

FIGURE 1: 12-lead EKG showing ST elevation changes at V1-V3.

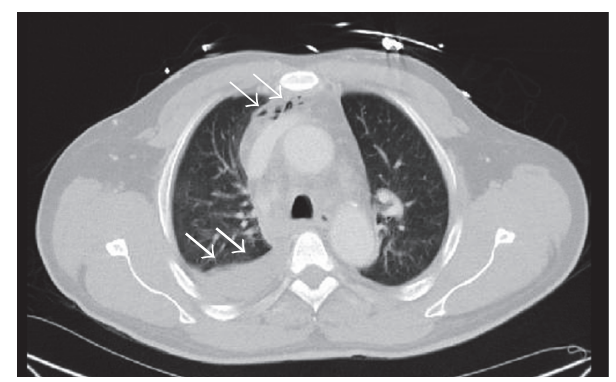

FIGURE 2: CT chest without contrast showed pneumomediastinum at the right superior mediastinum as shown by the white narrow arrow. The white thick arrow shows moderate right pleural effusion with 80 Hounsfield units suggestive of internal hemorrhage/proteinaceous content.

Coagulation profile done was within normal range with PT of 11.1 seconds (normal range 9.6-12.7), INR of 1.0 (normal range 0.9-1.1), and PTT of 30.0 (normal range 2.5-36.9). Serum electrolytes studies showed elevated serum potassium level $5.7 \mathrm{mEq} / \mathrm{L}$ (normal range $3.5-5.5 \mathrm{mEq} / \mathrm{L}$ ) with normal sodium, chloride, calcium, and bicarbonate levels.

Renal function tests showed elevated BUN $39 \mathrm{mg} / \mathrm{dL}$ (normal range 5-21 mg) and serum creatinine of $9.41 \mathrm{mg} / \mathrm{dL}$ (normal range $0.60-1.20 \mathrm{mg} / \mathrm{dL}$ ) with severely decreased estimated GFR $6 \mathrm{~mL} / \mathrm{min} / 1.73 \mathrm{~m}^{2}$ (normal > $60 \mathrm{~mL} / \mathrm{min} / 1.73 \mathrm{~m}^{2}$ ). Liver function tests showed elevated serum alkaline phosphatase 109 U/L (normal range 25$100 \mathrm{U} / \mathrm{L}$ ) with normal serum ALT and AST.

Initial Troponin ${ }^{-}$I done was $0.08 \mathrm{ng} / \mathrm{mL}$ (normal: $0.04-$ $0.80 \mathrm{ng} / \mathrm{mL}$ ). Electrocardiogram (Figure 1) showed normal sinus rhythm, normal axis, and presence of ST elevations in I, aVL, and V1-V4. Also noted were ST depressions in III, aVF, and V6 and incomplete LBBB.

Chest X-ray revealed a small right lower lobe infiltrate with subpulmonic effusion. Echocardiography revealed mild concentric left ventricular hypertrophy, impaired LV relaxation with abnormal (paradoxical) septal motion consistent with LBBB, noncompaction of ventricular apex, mild aortic regurgitation, and mitral regurgitation.

CT scan of chest revealed pneumomediastinum with mediastinal hematoma as seen in Figure 2.

\section{Treatment, Outcome, and Follow-up}

The patient presented to emergency room with acute nonradiating midsternal chest pain with evolving changes in anterior leads in EKG and a CODE STEMI was called for emergent cardiac catheterization as myocardial infarction is the most common and life-threatening condition that was needed to be ruled out in his EKG with ST elevation. Patient received aspirin and ticagrelor prior to catheterization. Coronary angiography showed normal coronary arteries but with mild global hypokinesis of the left ventricle that was already known even 6 months prior to this admission. Patient continued to have chest pain which was unrelieved by Nitroglycerin, while serial troponins remained negative and hence computed tomography of chest was done which revealed pneumomediastinum with mediastinal hematoma, likely a consequence of his unsuccessful attempted thrombectomy of his right brachiocephalic pain. Patient received topical as well oral pain medications for symptomatic relief. He did not require any surgical intervention as there was no collapsed lung and the volume of the pneumomediastinum can be absorbed by the body. He remained hemodynamically stable and was eventually discharged home with further follow-up with vascular surgery.

\section{Discussion}

Chest pain accounts for around 6 million annual visits to emergency department (ED) in the United States (US). Patients with EKG changes, characteristic symptoms, risk factors, and strong family history undergo emergent cardiac catheterization. However, in around $26 \%$ patients who underwent coronary angiograms, coronary arteries were found to be normal. In those patients, the presence of other noncardiac etiologies of acute chest pain needs to be assessed [1-4].

Pneumomediastinum is a rare condition described as abnormal presence of air or any other gas in the mediastinum. It can be spontaneous (primary) or secondary. Spontaneous pneumomediastinum is usually due to either infection or underlying lung conditions such as COPD or asthma [5]. Activities that cause increase in intrathoracic pressure such as Valsalva, coughing, and vomiting have also been reported to cause cases of spontaneous pneumomediastinum [6]. Secondary pneumomediastinum is usually a result of anatomic manipulation of mediastinal structures ranging from dental/endoscopic procedures [7], cardiac catheterizations, and endotracheal intubations to surgical interventions involving the thorax and abdomen. Chest or neck trauma causing tracheobronchial tree disruption has been reported to cause severe secondary tension pneumomediastinum. Pneumomediastinum secondary to injury of major venous vasculature at the time of central line placement has also been reported [8]. We present our case of secondary pneumomediastinum which resulted due to the manipulation of the right brachiocephalic vein, mimicking the signs and symptoms of a STEMI.

Apart from chest pain, patients with pneumomediastinum may also present with cough, shortness of breath, or changes in voice. Examination may be needed with 
Hamman's crunch or crepitations along the suprasternal notch. In terms of ECG findings, pneumomediastinum presents in various ways such as electrical alternans, T-wave inversions, poor R-wave progression, and low voltage QRS other than ST elevation $[9,10]$.

This is a rare case of secondary pneumomediastinum mimicking STEMI. The presence of other causes of chest pain in patients with normal coronary angiograms should be investigated. Care should be taken to ensure detailed history taking in regard to recent procedures involving the neck and thorax.

\section{Additional Points}

Learning Points/Take-Home Messages. In patients who have persistent chest pain with normal coronary arteries with cardiac catheterization, history of any thoracic invasive procedures should give rise to suspicion for pneumomediastinum as a differential diagnosis in patients who present with EKG finding of ST elevation.

\section{Disclosure}

The authors certify that they have no affiliations with or involvement in any organization or entity with any financial interest (such as honoraria, educational grants, participation in speakers' bureaus, membership, employment, consultancies, stock ownership, or other equity interest and expert testimony or patent-licensing arrangements) or nonfinancial interest (such as personal or professional relationships, affiliations, knowledge, or beliefs) in the subject matter or materials discussed in this manuscript.

\section{Conflicts of Interest}

The authors declare that they have no conflicts of interest.

\section{References}

[1] P. Widimsky, B. Stellova, L. Groch et al., "Prevalence of normal coronary angiography in the acute phase of suspected STelevation myocardial infarction: experience from the PRAGUE studies," Canadian Journal of Cardiology, vol. 22, no. 13, pp. 11471152, 2006.

[2] Y. L. Gu, T. Svilaas, I. C. C. Van Der Horst, and F. Zijlstra, "Conditions mimicking acute ST-segment elevation myocardial infarction in patients referred for primary percutaneous coronary intervention," Netherlands Heart Journal, vol. 16, no. 10, pp. 325-331, 2008.

[3] J. Brywczynski, J. McKinney, A. Brown, and C. Slovis, "Discerning STEMI mimics in the field," Journal of Emergency Medical Services, vol. 38, no. 12, 2013.

[4] W. C. Sin, J. M. Kwong, T. C. L. Wong, C. Kwong, C. Chan, and C. W. Siu, "Post-operative ST-segment elevation: not a blocked coronary artery, then what?" Cardiology Plus, vol. 1, no. 1, pp. 45-46, 2016.

[5] K. Keskin, M. Baskurt, F. Aktürk, and C. Conbayir, "A rare cause of chest pain mimicking myocardial infarction," Turk Kardiyoloji Dernegi Arsivi, vol. 42, no. 5, pp. 472-474, 2014.
[6] W. L. Dajer-Fadel, R. ArgÜero-Sánchez, C. Ibarra-Pérez, and F. P. Navarro-Reynoso, "Systematic review of spontaneous pneumomediastinum: a surgery of 22 years' data," Asian Cardiovascular \& Thoracic Annals, vol. 22, no. 8, pp. 997-1002, 2014.

[7] N. N. Johnson, A. Toledo, and E. E. Endom, "Pneumothorax, pneumomediastinum, and pulmonary embolism," Pediatric Clinics of North America, vol. 57, no. 6, pp. 1357-1383, 2010.

[8] Y. Wang, L. He, X. Hu, and Z. Zhou, "Inadvertent late migration of jugular catheter and bilateral pleural effusion and pneumomediastinum," International Journal of Critical Care and Emergency Medicine, vol. 2, no. 1, 2016.

[9] W. D. Brearley Jr., L. Taylor III, M. W. Haley, and L. Littmann, "Pneumomediastinum mimicking acute ST-segment elevation myocardial infarction," International Journal of Cardiology, vol. 117, no. 2, pp. e73-e75, 2007.

[10] R. M. Brandão, A. C. M. A. GonÇalves, R. P. M. Brandão, L. F. d Oliveira, A. C. Nogueira, and V. S. Kawabata, "Transient elevation of ST-segment due to pneumothorax and pneumopericardium," Autopsy and Case Reports, vol. 3, no. 1, pp. 6366, 2013. 


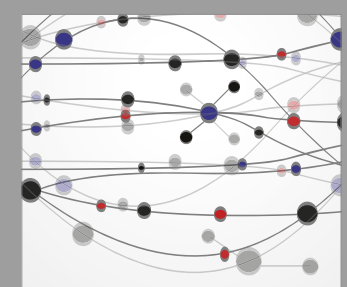

The Scientific World Journal
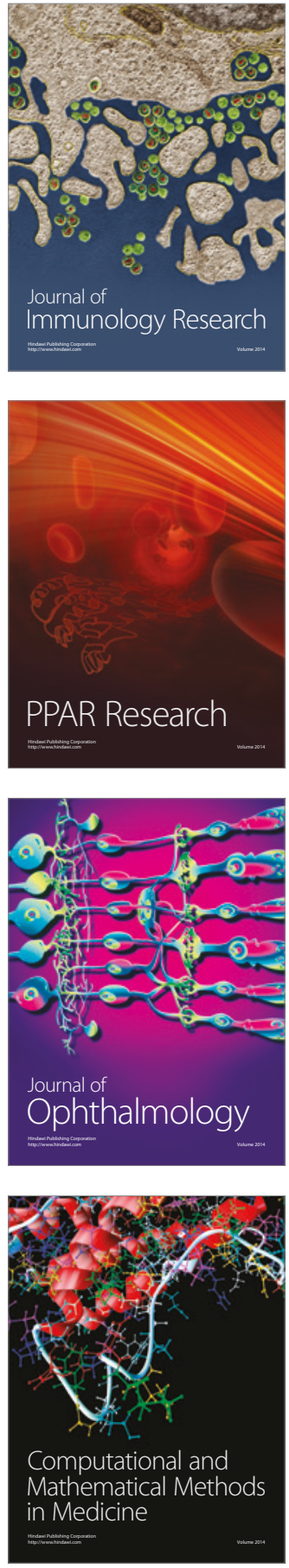

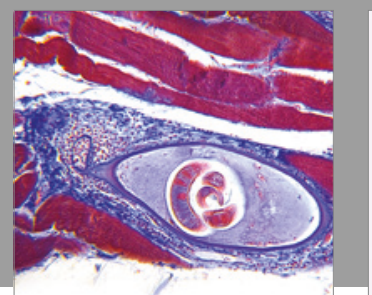

Gastroenterology Research and Practice
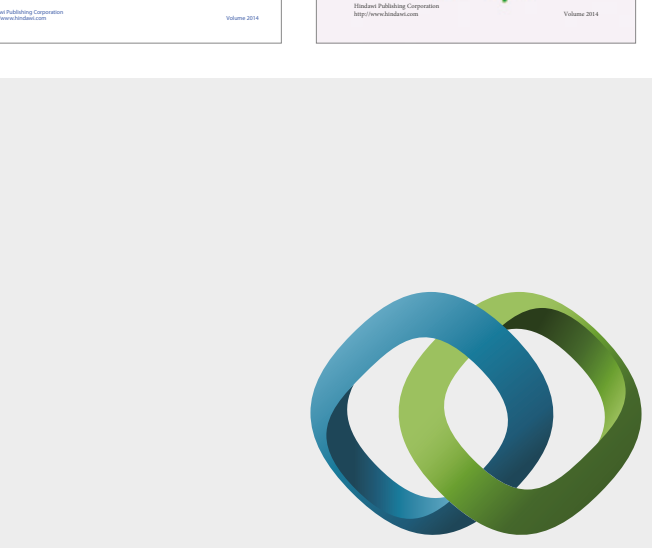

\section{Hindawi}

Submit your manuscripts at

https://www.hindawi.com
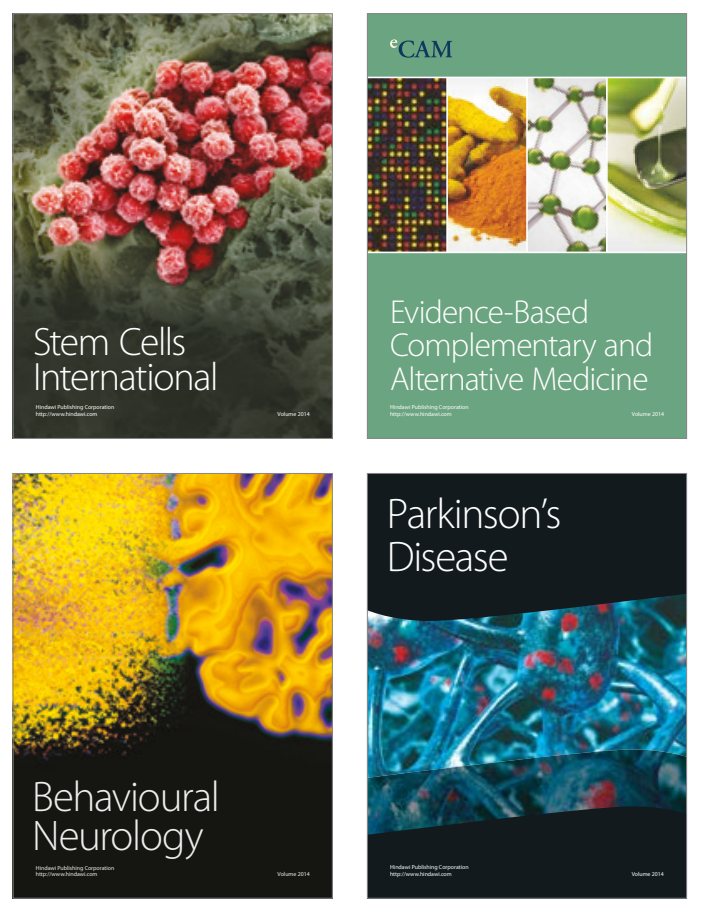
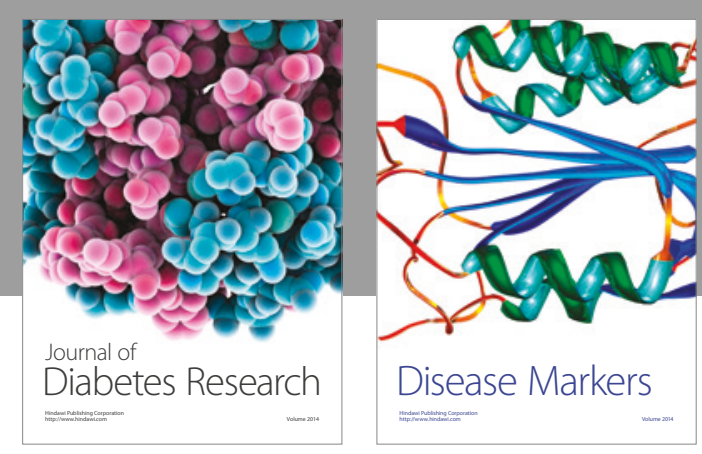

Disease Markers
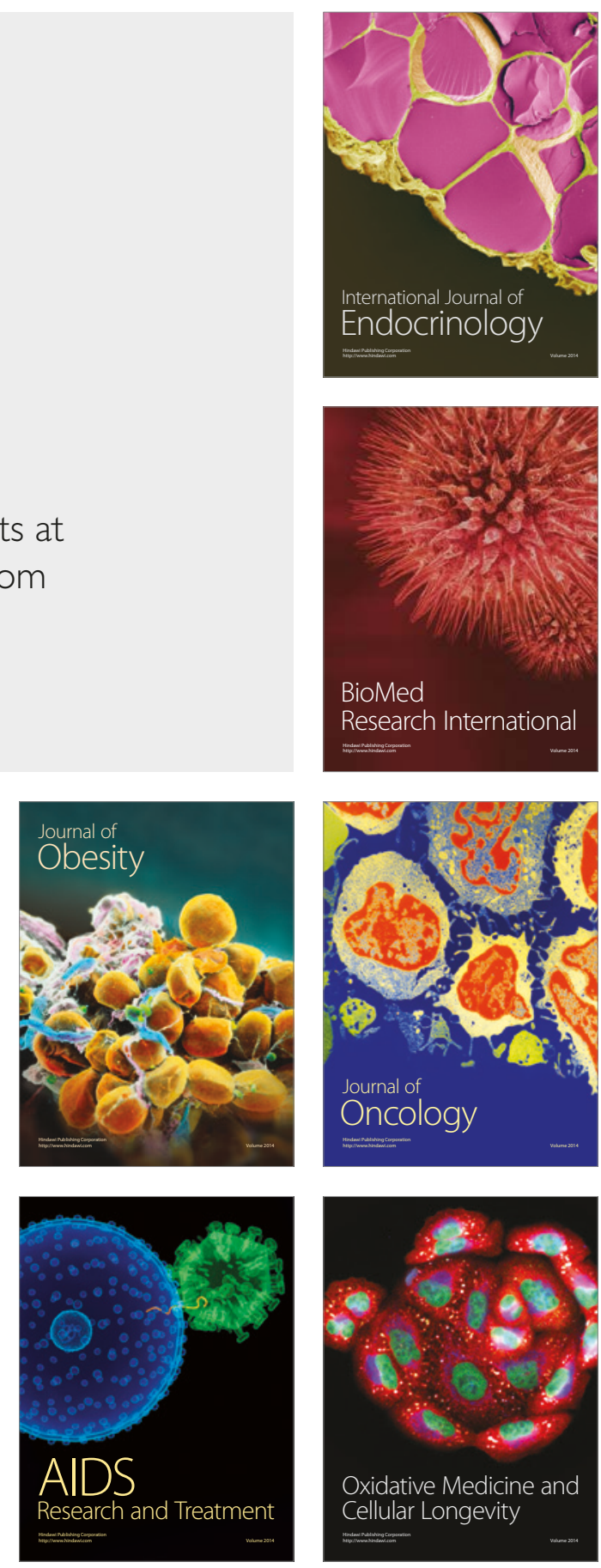